\title{
Prevalence of asthma-like symptoms and assessment of lung function in schoolchildren born with low birth weight
}

\author{
Nik Zainal ${ }^{1}$, mMed, Andy $\underline{\text { Rahardja }}^{2}$, mMed, Che Yusoff Faris Irfan $^{1}$, Msc, Ariffin Nasir $^{1}$, Mmed, \\ Wan Ibrahim Wan Pauzi ${ }^{1}$, MMed, Ilias Mohamad Ikram ${ }^{1}$, MMed, Hans Van Rostenberghe ${ }^{1}$, MD
}

INTRODUCTION This study aimed to determine the prevalence of asthma-like symptoms among schoolchildren with low birth weight (LBW), and to compare the lung function of these children with that of children with normal birth weight. METHODS This was a comparative cross-sectional study. We recruited children aged 8-11 years from eight primary schools in Kota Bharu, Kelantan, Malaysia. The children were divided into two groups: those with LBW (<2,500 g) and those with normal birth weight $(\geq 2,500 \mathrm{~g})$. Parents of the enrolled children were asked to complete a translated version of the International Study of Asthma and Allergies in Childhood questionnaire. Lung function tests, done using a MicroLoop Spirometer, were performed for the children in both groups by a single investigator who was blinded to the children's birth weight.

RESULTS The prevalence of 'ever wheezed' among the children with LBW was $12.9 \%$. This value was significantly higher than that of the children with normal birth weight (7.8\%). Forced vital capacity (FVC), forced expiratory volume in one second, and forced expiratory flow when $50 \%$ and $75 \%$ of the FVC had been exhaled were significantly lower among the children with LBW as compared to the children with normal birth weight.

CONCLUSION LBW is associated with an increased prevalence of asthma-like symptoms and impaired lung function indices later in life. Children born with LBW may need additional follow-up so that future respiratory problems can be detected early.

Keywords: asthma, child, low birth weight, lung function test, prevalence

\section{INTRODUCTION}

Low birth weight (LBW) has been defined by the World Health Organization as a weight of less than 2,500 g at birth. ${ }^{(1)}$ LBW has been reported to be associated with reduced lung function and respiratory illness in children. ${ }^{(2,3)}$ It has also been associated with lower lung function in adults, although symptoms of wheezing were not reported in adults. ${ }^{(4)}$ Although a few studies have found evidence that LBW is associated with asthma in children, ${ }^{(5,6)}$ other studies did not observe such an association. ${ }^{(7,8)}$ These findings seem to indicate some relationship between LBW and lung function; however, there is less consensus concerning the relationship between birth weight and respiratory symptoms.

About $10 \%$ of Malaysian children are born with LBW. ${ }^{(9)}$ At the time of writing, there was a lack of available data on the outcome of LBW infants in Malaysia or their health status when they reached childhood or adolescence. Hence, this study was conducted to investigate the prevalence of asthma-like symptoms among schoolchildren who had LBW, as well as to compare the lung function of schoolchildren who had LBW with that of schoolchildren who were born with normal weight.

\section{METHODS}

This was a comparative cross-sectional study. Approval for the study was obtained from the Education Department of Kelantan, Malaysia, and the Research and Ethics Committee of the School of Medical Sciences, Universiti Sains Malaysia, Malaysia.
We recruited children aged 8-11 years from eight primary schools in Kota Bharu, Kelantan, Malaysia. Random sampling with Microsoft Excel (Microsoft, Redmond, WA, USA) was used to select these eight schools from the 96 primary schools in Kota Bharu. ${ }^{(10)}$ Letters explaining the purpose of the study, along with a form for written consent, were distributed by class teachers to the parents of children aged 8-11 years in the eight schools. In Malaysia, schools keep a copy of the child's 'home-based immunisation card', which contains basic health information including birth weight, details on infant development and immunisation records. Schools regularly request for these records from parents and keep copies as part of their database of records.

To identify the children with LBW and those with normal birth weight, we reviewed the database of records of the eight schools. Children who were born with weight $<2,500 \mathrm{~g}$ at birth were included in this study, and made up the LBW group. Children who were born with weight $\geq 2,500 \mathrm{~g}$ at birth were randomly selected from class lists and invited to be included in the study; those who agreed to participate made up the normal birth weight group, which also served as the control group. Based on the children's medical history and notes in the school's database of records, children with obvious clinical syndromes, congenital malformations or a history of neonatal complications that required intensive care treatment were identified and excluded from the study.

${ }^{1}$ Department of Paediatrics, Universiti Sains Malaysia, Kelantan, ${ }^{2}$ Department of Paediatrics, Faculty of Medical and Health Sciences, Universiti Malaysia Sarawak, Sarawak, Malaysia Correspondence: A/Prof Nik Zainal Abidin Nik Ismail, Department of Paediatrics, Universiti Sains Malaysia, 16150 Kubang Kerian, Kelantan, Malaysia. nzainal@usm.my 
Parents of children from both groups were requested to complete a Malay version of the International Study of Asthma and Allergies in Childhood (ISAAC) questionnaire and to give written consent for their children to undergo assessment for spirometric measurements. The questionnaires and informed consent forms were distributed to the parents by school staff. The Malay version of the ISAAC questionnaire had been linguistically validated using backward and forward translations in a previous study. ${ }^{(11)}$ The questionnaire focuses on past and current wheezing episodes, the frequency of wheezing attacks in the previous 12 months, sleep disturbance, speech limitation during a wheezing attack, exercise-induced wheezing and nocturnal cough.

Children who had their parents' consent and also agreed to undergo spirometric measurements were invited to have the measurements done at the Paediatric Clinic of Hospital Universiti Sains Malaysia, Malaysia. Additional basic baseline data (e.g. current weight and height, and parental socioeconomic status) were collected during the clinic visit. Spirometry was conducted during school hours; during the measurements, the children were in their school uniforms and did not have shoes on. One of the eight schools that were randomly selected declined to participate in the spirometric measurements, as it only had three children aged 8-11 years who were born with LBW and they were occupied with school examinations during the study period. Weight was measured to the nearest $100 \mathrm{~g}$ and height was measured to the nearest $1 \mathrm{~cm}$. The lung function assessment was performed using the MicroLoop Spirometer SPIDA 5 (Micromedical, Rochester, Kent, England, UK). The following parameters were measured: forced vital capacity (FVC); forced expiratory volume in one second (FEV1); and forced expiratory flow when $25 \%, 50 \%$ or $75 \%$ of the FVC had been exhaled (FEF25, FEF50 and FEF75, respectively). A spirometry programme that employed an animated incentive (i.e. soap bubbles) was used to facilitate the testing.

The lung function assessment (i.e. spirometric measurements) were performed by a trainee paediatrician who was blinded to the children's birth weight. The procedure for performing the FVC manoeuvre was demonstrated to the children and measurements were only taken after three practice blows. The children were first told to relax, and then to put their lips around the mouthpiece (at this step, the paediatrician would check that the child's mouth fully covered the mouthpiece) and continue breathing normally. The children were then asked to inhale as deeply as possible and to exhale all the air from their lungs as quickly and forcefully as possible until no more air could be expelled from the lungs. A minimum of three manoeuvres were performed, which were recorded when they met the acceptability and repeatability criteria. (In this study, repeatability is defined as an FVC that is within $10 \%$ of the maximal FVC.) The children were encouraged to perform the tests to the best of their ability and at least 30 seconds of rest was given between each manoeuvre. Measurements were taken with the children in a standing position and without the use of a nose clip. The data was recorded in the Spida Spirometry Database system and analysed using SPSS version 12.0 (SPSS Inc, Chicago, IL, USA). Graphs that showed hesitancy or cough were not used in the analysis. For each child, the recording that showed the largest FVC and FEV1 was used for analysis.

Calculations of the required sample size showed that about 800 children were needed to achieve $80 \%$ power in our study on the prevalence of asthma-like symptoms among children with LBW. The calculations also suggested that about 400 children with LBW and normal birth weight were needed to compare the spirometric measurements (i.e. FVC, FEV1, FEF50 and FEF75) of the two groups of children, so that there would be at least $80 \%$ power to detect a difference of 0.1 unit in the spirometric measurements between the two groups.

Prevalence was expressed as percentage, while continuous data was expressed as mean \pm standard deviation. Normal distribution was checked for the continuous variables (i.e. weight, height and lung function indices). Independent $t$-test, chi-square test or Fisher's exact test were used to compare lung indices and asthma-like symptoms between the children with LBW and those with normal birth weight. A p-value $<0.05$ was considered statistically significant.

\section{RESULTS}

There were 807 children aged 8-11 years in the eight selected primary schools. The ISAAC questionnaires were distributed to the parents of these children and the parents of 770 children responded (response rate $=95.4 \%$ ). The parents of 217 children who had LBW and 203 children who had normal birth weight gave consent for their children to participate in the spirometric measurements. However, ten children from the LBW group did not turn up for the spirometric and baseline data measurements at the paediatric clinic. Hence, a total of 207 children who had LBW underwent spirometric measurements. All 203 children with normal birth weight turned up for the measurements. The baseline demographic data of the children who underwent spirometric measurements is shown in Table I. Other than current weight, both the LBW group and normal birth weight group showed similar baseline characteristic data.

Prevalent asthma-like symptoms, based on the responses gathered in the questionnaires, are shown in Table II. The prevalence of 'ever wheezed' in the LBW group was significantly higher than that in the normal birth weight group $(12.9 \%$ vs. $7.8 \%, p=0.03)$. Children in the LBW group also had higher prevalence of night cough in the last 12 months than those in the normal birth weight group $(20.3 \%$ vs. $15.2 \%)$, but this was not statistically significant $(p=0.09)$.

Table III shows the lung function indices of the LBW normal birth weight groups. Most spirometric measurements (i.e. FVC, FEV1, FEF50 and FEF75) were significantly lower in the LBW group than in the normal birth weight group. No significant difference was detected between the two groups for only FEF25.

\section{DISCUSSION}

The present study found that there was a higher prevalence of 'ever wheezed' among schoolchildren born with LBW than 
Table I. Baseline characteristics of the children who underwent spirometric measurements $(n=410)$.

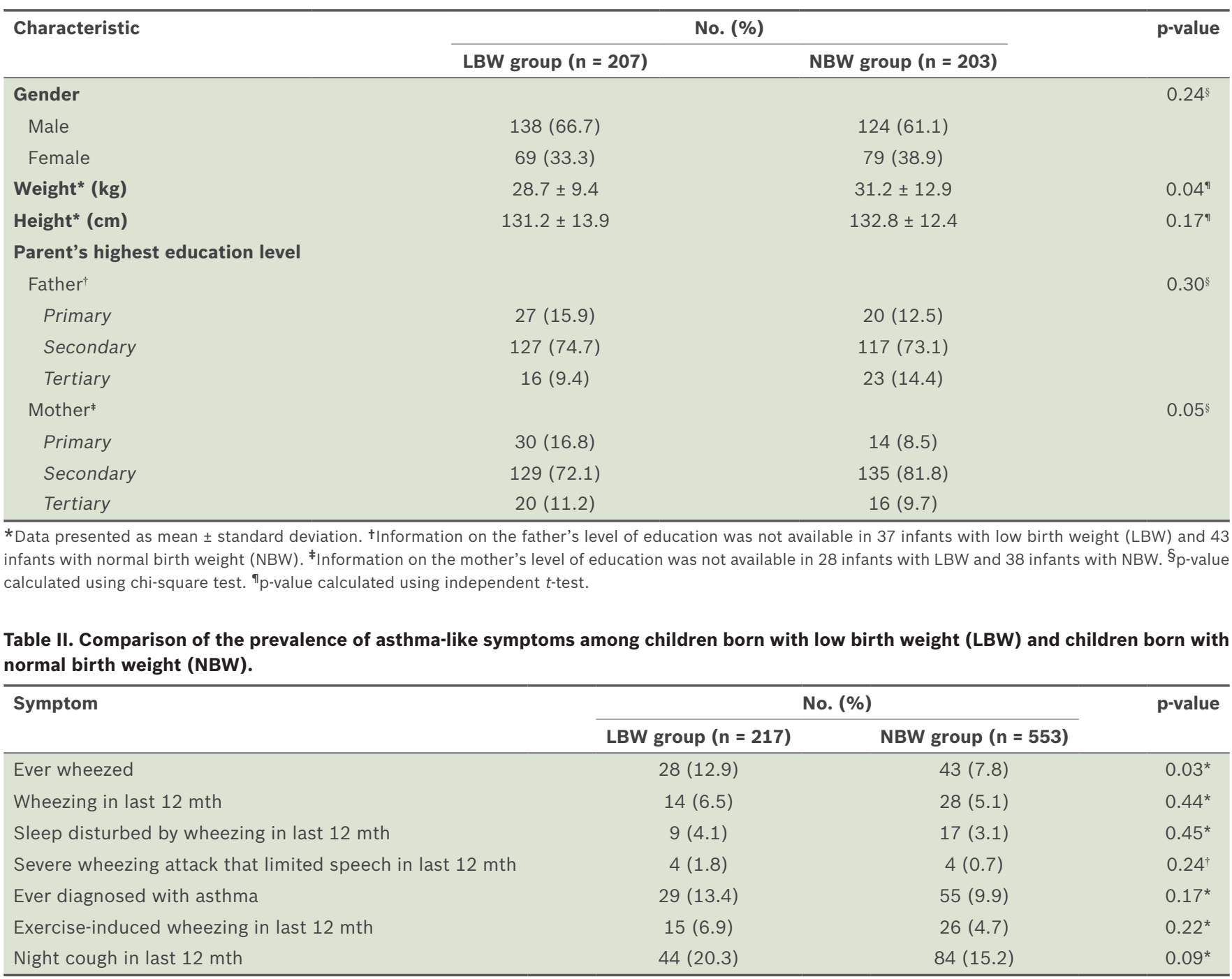

p-value calculated using * chi-square test; †Fisher's exact test.

Table III. Lung function indices in children born with low birth weight (LBW) or normal birth weight (NBW).

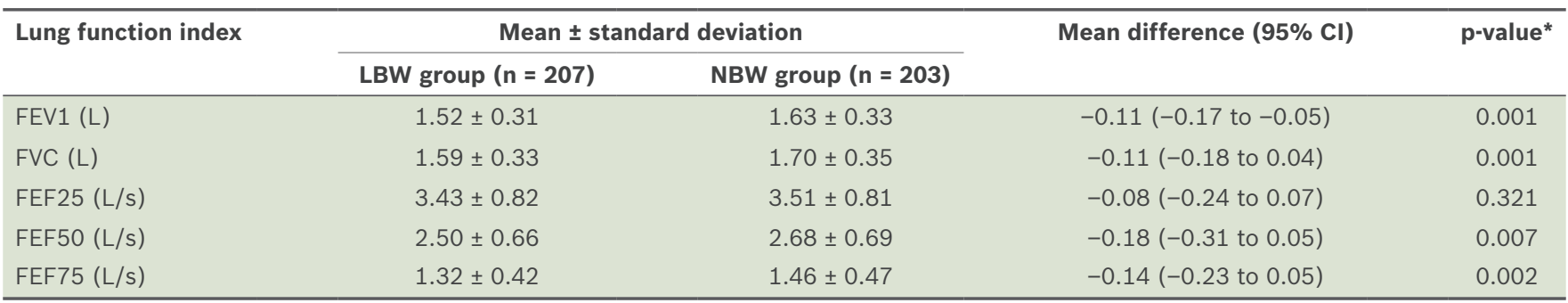

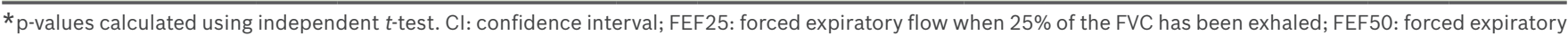
flow when $50 \%$ of the FVC has been exhaled; FEF75: forced expiratory flow when $75 \%$ of the FVC has been exhaled; FEV1: forced expiratory volume in 1 second; FVC: forced vital capacity.

among schoolchildren born with normal birth weight $(12.9 \%$ vs. $7.8 \%$ ). This finding is similar to that of a study conducted by Brooks et al, which found that the prevalence of 'ever wheezed' among LBW children was $10.9 \%$, while that of children born with normal birth weight was $6.7 \%{ }^{(12)}$ The prevalence of 'ever wheezed', wheezing in the last 12 months, and 'ever diagnosed with asthma' in the LBW group of the present study $(12.9 \%, 6.5 \%$ and $13.4 \%$, respectively) were higher than those reported in a previous study of schoolchildren conducted by Quah et al in Kota Bharu $\left(9.4 \%, 6.0 \%\right.$ and $9.4 \%$, respectively). ${ }^{(11)}$
The findings of the present study suggest that infants born with LBW are at risk of having decreased lung function during childhood. In our study, we found that children who were born with LBW had significantly lower FVC, FEV1 and FEF than children with normal birth weight. This indicates that these children may have reduced lung size, and large and small airway obstructions. A reduced lung size and the presence of obstructions may be possible reasons for the increased prevalence of wheezing among these children. These findings are similar to those of the EPICure study ${ }^{(13)}$ and the study by Cazzato et al. ${ }^{(14)}$ However, 
those two studies involved children who were extremely preterm or had very LBW (i.e. $<1,500$ g). ${ }^{(13,14)}$

In the present study, we excluded children who had a history of obvious clinical syndromes, such as problems with ventilation, as we wanted to reduce potential confounders and aimed to examine the impact of birth weight on respiratory symptoms. It is important to note that wheezing and nocturnal cough may not necessarily be due to asthma; these respiratory symptoms may occur in other diseases as well. Future studies should include parameters such as the measurement of tobacco smoke exposure and gestational age, and include physical examinations to look for evidence of chest deformities, such as pectus excavatum.

In conclusion, LBW infants are at a higher risk of having asthma-like symptoms, such as wheezing and nocturnal cough, and impaired lung function later in life as compared to infants born with normal weight. Further research is necessary to improve our understanding of the factors that predispose LBW infants to having asthma-like symptoms and reduced lung function later in life.

\section{REFERENCES}

1. World Health Organization. International Statistical Classification of Diseases and Related Health Problems. World Health Organization, Geneva, 1992.

2. Chan KN, Elliman A, Bryan E, Silverman M. Respiratory symptoms in children of LBW. Arch Dis Child 1989; 64:1294-304.
3. Chan KN, Noble-Jamieson CM, Elliman A, Bryan EM, Silverman M. Lung function in children of LBW. Arch Dis Child 1989; 64:1284-93.

4. Barker DJ, Godfrey KM, Fall C, et al. Relation of birth weight and childhood respiratory infection to adult lung function and death from chronic obstructive airways disease. BMJ 1991; 303:671-5.

5. Schwartz J, Gold D, Dockery DW, Weiss ST, Speizer FE. Predictors of asthma and persistent wheeze in a national sample of children in the United States. Association with social class, perinatal events, and race. Am Rev Respir Dis 1990; 142:555-62.

6. Weitzman M, Gortmaker S, Sobol A. Racial, social, and environmental risks for childhood asthma. Am J Dis Child 1990; 144:1189-94.

7. Sears MR, Holdaway MD, Flannery EM, Herbison GP, Silva PA. Parental and neonatal risk factors for atopy, airway hyper-responsiveness, and asthma. Arch Dis Child 1996; 75:392-8.

8. Fergusson DM, Crane J, Beasley R, Horwood LJ. Perinatal factors and atopic disease in childhood. Clin Exp Allergy 1997; 27:1394-401.

9. Wardlaw T, Blanc A, Zupan J, Ahman E. United Nations Children's Fund and World Health Organization. Low Birthweight: Country, regional and global estimates. UNICEF: New York, 2004.

10. Naing L. Single Proportion Calculator Version 1.0. Available at: http:// www.kck.usm.my/ppsg/stats_resources.htm. Accessed February 15, 2006.

11. Quah BS, Razak AR, Hassan MH. Prevalence of asthma, rhinitis and eczema among schoolchildren in Kelantan, Malaysia. Acta Paediatr Jpn 1997; 39:329-35.

12. Brooks AM, Byrd RS, Weitzman M, Auinger P, McBride JT. Impact of LBW on early childhood asthma in the United States. Arch Pediatr Adolesc Med 2001; 155:401-6.

13. Fawke J, Lum S, Kirkby J, et al. Lung function and respiratory symptoms at 11 years in children born extremely preterm: the EPICure study. Am J Respir Crit Care Med 2010; 182:237-45.

14. Cazzato S, Ridolfi L, Bernardi F, Faldella G, Bertelli L. Lung function outcome at school age in very LBW children. Pediatr Pulmonol 2013; 48:830-7. 